\title{
The action of light on culture media
}

\author{
PAMELA M. WATERWORTH \\ From the Department of Bacteriology, Royal Postgraduate Medical School, London
}

SYNOPSIS Failure of growth of staphylococci on nutrient agar plates was shown to be due to previous exposure to sunlight in an exceptionally well lit laboratory. Such plates inhibited the growth of Staph. aureus, Staph. albus, some Proteus species and Providence, but not that of various other bacteria. The affected species are found to be more susceptible to the action of hydrogen eroxide added to the medium.

The effect was produced in plates of five different nutrient agars, but not in two others which contained casein.

Plates awaiting inoculation should be protected from light; a white fabric blind was found not to be fully protective.

This study was prompted by failure to grow staphylococci on nutrient agar. Sensitivity tests on nutrient agar plates inoculated by flooding with a suspension of Staph. aureus diluted to give dense but not confluent growth either failed to grow at all, gave only a few isolated colonies, or sometimes an area of near normal growth on one side of the plate (Fig. 1a). Further incubation with or without re-inoculation did not produce significant growth, though 'satellitism' occurred round existing colonies. This problem had recurred intermittently over a period of 18 months but all attempts to reproduce it deliberately had failed.

In January 1968 in an experiment to show the effect of bacterial growth on the $p \mathrm{H}$ of the medium, small areas of a filter membrane applied to nutrient agar plates were heavily inoculated with staphylococci. After overnight incubation the membrane was removed and the plate flooded with a dilute suspension of the Oxford staphylococcus. Growth appeared only on areas over which there had been growth on the membrane.

During the following fortnight plates were frequently inhibitory and possible explanations for this effect which were examined and disproved were: a defect in the medium itself (Oxoid blood agar base no. 2); the presence of an inhibitory substance in the water with which it was made up; the presence of such a substance in the water used for diluting the bacterial suspension; undue drying of the medium surface; the liberation of a substance interfering with growth from the plastic Petri dishes or Pasteur pipettes used. All strains of Staph. aureus seemed equally susceptible and on one occasion, Proteus mirabilis was similarly inhibited. It was suggested that hydrogen peroxide might be formed during excessive heating of the medium. This was shown not to be the explanation but it was found that when cups containing catalase were added to inhibitory plates, growth occurred in the surrounding area. The clue to the true explanation was found in the following sentence (Elek, 1959): 'Coagulase-positive staphylococci exhibit catalase activity. Burnet (1925) first demonstrated this enzyme when he observed a diffusible product of Staph. aureus which destroyed hydrogen peroxide formed by exposure of nutrient agar plates to sunlight or a carbon arc.'

\section{EXPERIMENTAL OBSERVATIONS}

The Bacteriology Department of this School moved to the eighth floor of the Commonwealth Building in June 1966 and the effects described above had been seen only since that date. The eighth floor is 92 feet (28 metres) above ground level. The length of this building is aligned approximately WNWESE, and the laboratory in which the work was done occupies the SE corner, with windows on both external walls, ie, facing approximately $\mathrm{E}$ and $\mathrm{S}$. Moreover they are large windows extending from 9 in. $(22.86 \mathrm{~cm})$ from above the bench to the ceiling $5 \mathrm{ft} 4 \mathrm{in} .(1 \cdot 625 \mathrm{~m})$ above and the full width of each wall, that on the $E$ being $12 \mathrm{ft} 11$ in. $(3.937 \mathrm{~m})$ wide and that on the $S 8 \mathrm{ft} 7 \mathrm{in}$. $(2 \cdot 616 \mathrm{~m})$. The remaining two walls are white. This is thus probably the most sunlit bacteriological laboratory in London, if not in the whole of Great Britain. This has been found a drawback for the purpose of microscopy, but the 
generous illumination in the clearer upper air of the metropolis has been found to favour the luxuriant growth of exotic plants in other rooms facing $\mathrm{E}$ or $\mathrm{S}$.

MATERIALS AND METHODS In all experiments involving exposure of medium to direct light, plates were placed inverted on the $\mathbf{S}$ window sill (unless otherwise stated). As both the intensity and constancy of sunshine vary, plates of Oxoid blood agar base no. 2 (BAB 2) were included in all tests as a standard. All plates contained $15 \mathrm{ml}$ medium giving a depth of $3 \mathrm{~mm}$. Unless otherwise stated all were inoculated by flooding with a broth culture of the Oxford Staph. aureus diluted to give dense but not confluent growth on the control plate. Results were recorded as total inhibition (no growth or a small number of isolated colonies) and partial inhibition (number and/or size of colonies reduced). It was sometimes found that only part of the plate was affected and the area showing most inhibition was then recorded.

\section{RESULTS}

EFFECT ON SUBSEQUENT GROWTH OF EXPOSING MEDIA TO LIGHT The length of exposure to light required to render the medium inhibitory varied in different experiments, but even as early in the year as 1 February, plates of BAB2 exposed to direct sunlight were partially inhibitory after $15 \mathrm{~min}$ and totally after $25 \mathrm{~min}$. Plates left on the bench out of the sun showed a slight reduction of growth after one and a half hours, marked inhibition at three and thereafter were sterile. Similarly plates left on the bench on a dull, cloudy day and those exposed in another room having only a north window all showed partial inhibition after three hours and were sterile in four.
Perhaps even more disturbing, plates left on the $\frac{0}{5}$ bench beneath the $\mathbf{S}$ window which had a white fabric blind down protecting them from direct sun $-\frac{\vec{F}}{5}$ light, were partially inhibitory after one hour and $\frac{5}{5}$ totally after one and a half hours.

Replica plating of some of the sterile plates con- $-\overline{\bar{\omega}}$. firmed that the inoculum had been killed.

EFFECT OF COMPOSITION OF MEDIUM Other com-๘๊ mercial nutrient agars, or nutrient broths with $1.25 \%$ agar added, were tested in the same way:Oxoid nutrient broth no. 2 (B2), Difco nutrient broth $\vec{\omega}$ (DNB) and Oxoid diagnostic sensitivity test agare (DST) all became inhibitory nearly as quickly aso̊ BAB2 and although both Oxoid and Difco brain N heart infusion (BHI) required longer periods of ex- $-\omega$ posure when first poured, these also became inhibitory when exposed after a few days' storage at $4{ }^{\circ} \mathrm{C} . \omega$ On the other hand, Mueller Hinton agar (MH) and? Oxoid tryptone soya broth (TSB) were unaffected by $\vec{r}$ three hours' exposure to bright sun even after storage. The addition of $10 \%$ serum did not prolong the time required to render $\mathrm{BAB} 2$ inhibitory, but theco addition of blood (whole, lysed, or heated) prevented. the effect altogether.

SUSCEPTIBILITY TO OTHER BACTERIA Similar tests using other organisms showed that BAB2 agaro quickly became inhibitory for $\operatorname{Pr}$. mirabilis, $\operatorname{Pr} . \frac{\mathrm{Q}}{\mathrm{D}}$ rettgeri, and $P r$. morgani and required only a little longer exposure to inhibit Providence. Growth of Esch. coli, Ps. aeruginosa, Str. faecalis, and Str. pyogenes was unimpaired after exposure of the plates to bright sun for three hours.
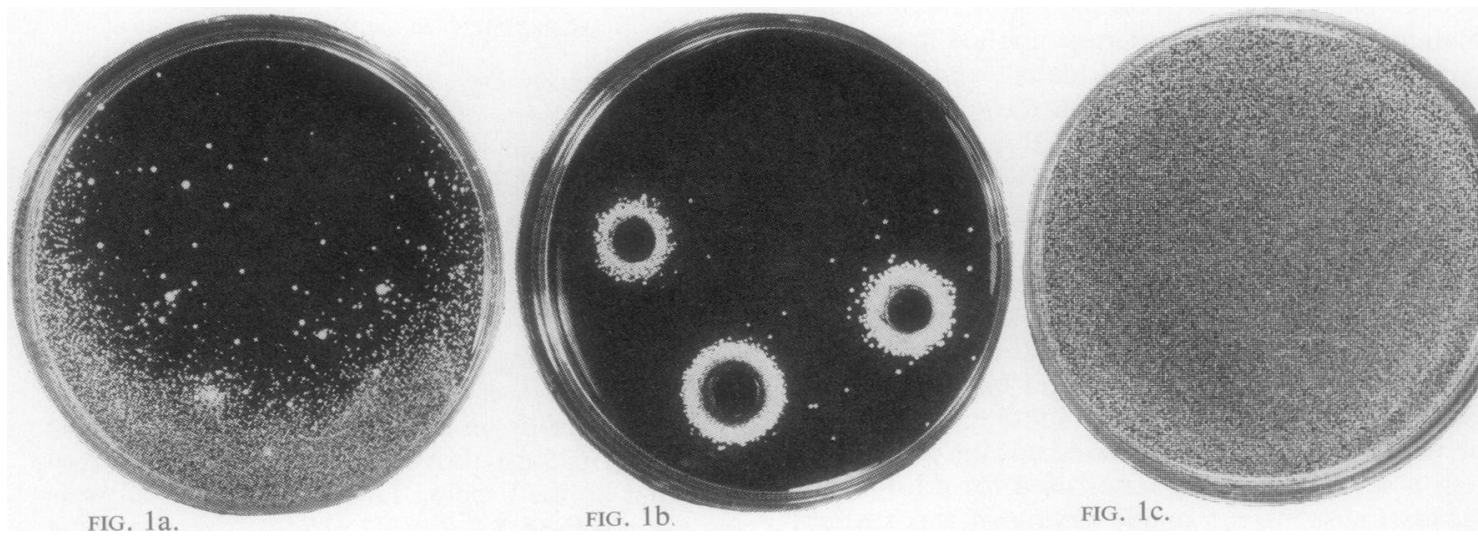

FIG. 1. Growth of a light inoculum of Staph. aureus on nutrient agar (BAB2): (a) Plate inoculated after briefo exposure to the sun. (b) Plate inoculated after slightly longer exposure. Cups contain (left to right) $1 / 50$ catalase $\mathrm{N} / 10 \mathrm{HCl}$, and $10 \%$ casein hydrolysate. (c) Control plate protected from the light throughout. 
NEUTRALIZATION OF INHIBITORY EFFECT Attempts were made to neutralize the inhibitory effect by adding in turn catalase, casein, and acid to the cups.

Catalase If the inhibition was due to the formation of hydrogen peroxide, the effect should be neutralized by the addition of catalase. When a cup containing 1/50 catalase (higher concentrations were themselves inhibitory) was added to BAB2 plates after varying exposures to the sun, a small zone of growth surrounded it when the medium was only just inhibitory, but not after more prolonged exposure. On the other hand, if $0.5 \%$ catalase was added to BAB2 when poured, the medium supported normal growth after three hours in the sun. When $0 \cdot 12 \%$ was added there was partial inhibition after two and a half hours and complete at three.

Casein The main difference between those media which are made toxic by light and those which are not is that the latter have casein as their source of nitrogen and do not contain commercial peptone. When $1.75 \%$ casein hydrolysate (as in $\mathrm{MH}$ ) or $1.7 \%$ tryptone (as in TSB) was added to BAB2, the medium supported normal growth after three hours' exposure to the sun. The addition of $0.3 \%$ soya peptone (also present in TSB) only prolonged the life of the medium by about one hour. If cups containing $10 \%$ solutions of any of these were added to exposed BAB2 plates, all produced a large zone of growth on plates which were just inhibitory, but not after prolonged exposure. Ten per cent peptone in cups had a similar but less pronounced effect. All these solutions were brought to $p H$ 7. The effect of catalase, casein, and acid is illustrated in Figure $1 \mathrm{~b}$.

Acid A chance observation early in this work suggested that a drop in $p \mathrm{H}$ reduced the inhibitory effect. It was found that the addition of $\mathrm{N} / 10 \mathrm{HCl}$ in a cup produced a large area of growth except when the exposure had been prolonged. When the $p \mathrm{H}$ of BAB2 was adjusted by the addition of hydrochloric acid or sodium hydroxide, plates at $p \mathrm{H} \mathrm{7.5,}$ 8.0 , and 8.5 all became inhibitory at the same rate, those at 7.0 and 6.5 required progressively longer and at 6.0 the medium supported normal growth after three hours' exposure to the sun.

EFFECT OF STORAGE Dieudonné (1894b) found that the hydrogen peroxide formed in medium soon disappeared if the plates were placed in the dark. In the present work it was found that plates which were only marginally inhibitory might subsequently support growth, but after longer exposures even three days at either $4^{\circ}$ or $37^{\circ} \mathrm{C}$ failed to permit growth. However, when cups containing casein, $\mathrm{N} / 10 \mathrm{HCl}$, and catalase were added to such plates, growth appeared round the first two but not round the catalase.
STUDIES WITH HYDROGEN PEROXIDE

SUSCEPTIBILITY OF DIFFERENT SPECIES Plates of BAB2 containing dilutions of hydrogen peroxide were lightly inoculated with multiple strains of different species. The results are given in Table I and show that the species which fail to grow on media which have been exposed to light are also more sensitive to hydrogen peroxide.

\section{TABLE I}

MINIMUM INHIBITORY CONCENTRATION OF HYDROGEN PEROXIDE FOR DIFFERENT SPECIES OF BACTERIA

\begin{tabular}{|c|c|c|c|c|c|}
\hline \multirow{2}{*}{$\begin{array}{l}\text { Strains } \\
\text { Tested }\end{array}$} & \multicolumn{5}{|c|}{ No. of Struins Inhibited } \\
\hline & $\begin{array}{l}1: \\
8,000\end{array}$ & $\begin{array}{l}1: \\
16,000\end{array}$ & $\begin{array}{l}1: \\
32,000\end{array}$ & $\begin{array}{l}1: \\
64,000\end{array}$ & $\begin{array}{l}1: \\
128,000\end{array}$ \\
\hline
\end{tabular}

\begin{tabular}{|c|c|c|c|c|c|c|}
\hline Staph. aureus & 35 & & & & 35 & \\
\hline Staph. albus & 6 & & & & 6 & \\
\hline Str. pyogenes & 8 & & 8 & & & \\
\hline Str. pneumoniae & 13 & 13 & & & & \\
\hline Str. viridans & 3 & 3 & & & & \\
\hline Str. faecalis & 10 & 9 & 1 & & & \\
\hline S. lutea & 1 & & & 1 & & \\
\hline L. casei & 1 & 1 & & & & \\
\hline Neisseria spp. & 7 & & 1 & 4 & 1 & 1 \\
\hline Esch. coli & 9 & & 9 & & & \\
\hline Klebsiella & 9 & & 6 & 3 & & \\
\hline Pr. mirabilis & 1 & & & 1 & & \\
\hline Pr. morgani & 5 & & & & 5 & \\
\hline Pr. rettgeri & 4 & & & 3 & 1 & \\
\hline Providence & 2 & & & 2 & & \\
\hline Ps. aeruginosa & 8 & & 8 & & & \\
\hline
\end{tabular}

EFFECT OF MEDIUM COMPOSITION Dilutions of hydrogen peroxide were prepared in plates of BAB2 and lightly inoculated with the Oxford staphylococcus. Growth was inhibited by 1 in 64,000 , but the addition of cups containing $10 \%$ peptone, $10 \%$ casein hydrolysate, $5 \%$ soya peptone, 1/50 catalase, and $\mathrm{N} / 10 \mathrm{HCl}$ all produced a large zone of growth on this dilution and a smaller zone on plates containing 1 in 32,000 . There was no growth on 1 in 16,000 . When the sensitivity of a variety of organisms to hydrogen peroxide was tested on BAB2 with or without $0.5 \%$ casein hydrolysate, there was a twofold increase in the MIC of Staph. aureus and Proteus spp. when casein was present but no change in that of Ps. aeruginosa, Esch. coli, or Klebsiella. When cups containing $10 \%$ casein, $\mathrm{N} / 10 \mathrm{HCl}$, and $1 / 50$ catalase were added to plates containing 1 in 16,000 hydrogen peroxide and inoculated with Esch. coli or $P$ s. pyocyanea, there was a small zone of growth round that containing catalase but not round the casein or acid.

PH The MIC for the Oxford staphylococcus and Esch. coli were also determined in BAB2 at different $p H$. That of the staphylococcus was found to be 1 in 


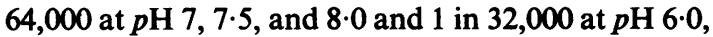
$6 \cdot 5$, and 8.5. The Esch. coli was inhibited by 1 in 16,000 throughout.

CATALASE The addition of $0.25 \%$ catalase produced a two- to four-fold increase in the MIC for multiple strains of staphylococci: $0.5 \%$ permitted normal growth on 1 in 2,000 (highest concentration tested).-

EFFECT OF HEATING Burnet (1925) found that heating to $100^{\circ} \mathrm{C}$ for $30 \mathrm{~min}$ removed the inhibitory effect unless exposure had been very prolonged. Dilutions of hydrogen peroxide were added to BAB2 in duplicate; one set was poured at once and the second left for two to three hours then heated to $100^{\circ} \mathrm{C}$ for $15 \mathrm{~min}$ and poured. When these plates were inoculated with Esch. coli the MIC rose from 1 in 16,000 before heating to 1 in 4,000 after. When the Oxford staphylococcus was tested in this way, the MIC remained 1 in 64,000 unless casein was added to the medium when it rose to 1 in 4,000 too. These results are given in Table II.

TABLE II

MINIMUM INHIBITORY CONCENTRATION OF HYDROGEN PEROXIDE BEFORE AND AFTER HEATING TO $100^{\circ} \mathrm{C}$ FOR $15 \mathrm{MIN}$

\begin{tabular}{|c|c|c|c|c|c|c|}
\hline & \multicolumn{4}{|c|}{ Oxford Staph. aureus } & \multicolumn{2}{|c|}{ Esch. coli } \\
\hline & \multicolumn{2}{|c|}{ Before Heating } & \multicolumn{2}{|c|}{ After Heating } & \multirow{2}{*}{$\begin{array}{l}\text { Before } \\
\text { Heating }\end{array}$} & \multirow{2}{*}{$\begin{array}{l}\text { After } \\
\text { Heating }\end{array}$} \\
\hline & $B A B 2$ & $\begin{array}{l}+1.5 \% \\
\text { Casein }\end{array}$ & $B A B 2$ & $\begin{array}{l}+1.5 \% \\
\text { Casein }\end{array}$ & & \\
\hline $1: 2,000$ & - & - & - & - & - & - \\
\hline $1: 4,000$ & - & - & - & - & - & - \\
\hline $1: 8,000$ & - & - & - & + & - & ++ \\
\hline $1: 16,000$ & - & - & - & ++ & - & ++ \\
\hline $1: 32,000$ & - & - & -- & ++ & ++ & ++ \\
\hline $1: 64,000$ & - & $+t$ & - & ++ & ++ & ++ \\
\hline $1: 128,000$ & ++ & ++ & $+t$ & ++ & ++ & ++ \\
\hline
\end{tabular}

\section{DISCUSSION}

Light can affect bacterial growth in three ways. That it can exert a direct bactericidal effect has been recognized since almost the earliest days of bacteriology. The first suggestion of an indirect effect was the observation of Roux (1887) that exposure to sunlight for three to four hours deprived broth of the capacity to support the growth of $B$. anthracis from a spore suspension. Dieudonné (1894b) provided the first explanation of this: he showed that nutrient agar or gelatin after exposure to sunlight contained hydrogen peroxide. Thirdly, Anderson and Cowan (1968) have recently shown that if bacto-folic acid casei medium (Difco no. 0822) is exposed to light it will no longer support the growth of $L$. casei. They found this to be due to the destruction of riboflavine.
This subject was again studied by Burnet (1925) who verified that hydrogen peroxide was responsible for the failure of small numbers of staphylococci to $\overrightarrow{\vec{s}}$ grow on nutrient agar plates which had been exposed to light. The present work has shown that this effect is greatly influenced by the composition of the medium and suggests that the action of low concentrations of hydrogen peroxide on staphylococcio may be of a different nature to that of higher con-s centrations on less sensitive organisms. No attempte has been made to measure the amount of hydrogenperoxide formed in plates exposed to light, but the complete protection afforded by casein and the failure to prevent the growth of Esch. coli indicate that it cannot be very high. Whilst the addition of catalase affects all organisms and clearly acts by neutralizing hydrogen peroxide, the addition of casein affects only those organisms which are most sensitive to hydrogen peroxide, and suggests that this greater sensitivity may be due to a nutritiona $\vec{z}$ deficiency.

As the $L$. case $i$ is one of the most resistant organ isms it seemed unlikely that the loss of vitamin Bo described by Anderson and Cowan (1968) was ${ }^{\circ}$ responsible and it was confirmed that the addition? of riboflavine to inhibitory medium did not permit growth of staphylococci. It seems more likely that amino acids are involved and on this basis the effecto of acid in cups might be explained by the possible hydrolysis of the peptone in the surrounding medium $\overrightarrow{\vec{F}}$

Although older textbooks (eg Browning ands Mackie, 1949) contain references to the action of light on culture media, those in use at the presento day are at least for the most part silent about it. A fresh warning about the exposure of culture media to light is therefore perhaps not out of place.

Admittedly the conditions in the laboratory where these observations were made are quite exceptional. On the other hand, there must be many others particularly in modern buildings with large windows? where plates may be exposed to strong light. Glase is reputed to filter off some of the bactericidal rays at the ultraviolet end of the spectrum, but it is clearo from many reported experiments that even two layers of glass, the window and the Petri dish, fail to prevent either the direct lethal effect or the formation of hydrogen peroxide. (These experiments were done in plastic dishes, but it was also found that the medium could be rendered inhibitory in a glass dish. D Moreover, although direct sunlight can exert such ${ }^{+}$ effects very quickly $(15 \mathrm{~min})$, diffuse daylight can produce it more slowly (three hours), and, according to Dieudonné (1894a), even electric light. It there $\frac{\text { P }}{?}$ fore seems advisable that, so far as is possible, nutrient agar plates should be protected from the light at all times. It may also be advisable to con 
sider the choice of medium for cross-infection studies using 'settle plates', which may be exposed for several hours in light wards. The addition of $0.01 \%$ phenylphthalein diphosphate to BAB2 did not affect the rate at which this medium became inhibitory.

I wish to thank Professor L. P. Garrod for his help in preparing this paper and for translating the papers by Dieudonné from the German.

\section{REFERENCES}

Anderson, B. B., and Cowan, J. D. (1968). J. clin. Path., 21, 85. Browning, C. H. and Mackie, T. J. (1949). Textbook of Bacteriology. Oxford University Press.

Burnet, F. M. (1925). Aust. J. exp. Biol. med. Sci., 2, 65.

Dieudonné, A. (1894a). Arb. Gesundh-Amte (Berl.), 9, 405.

(1894b). Ibid., 537.

Elek, S. (1959). Staphylococcus pyogenes and its Relation to Disease. Livingstone, Edinburgh.

Roux, E. (1887). Ann. Inst. Pasteur, 1, 445. 